\title{
BMJ Open Protocol for a qualitative study on promoting dietary change and positive food choices for poor people with low income who experience cardiovascular disease in Pakistan
}

\author{
Rubina Iqbal Barolia, Alexander M Clark, Gina M A Higginbottom
}

To cite: Barolia RI, Clark AM, Higginbottom GMA. Protocol for a qualitative study on promoting dietary change and positive food choices for poor people with low income who experience cardiovascular disease in Pakistan. BMJ Open 2013;3:e004176. doi:10.1136/ bmjopen-2013-004176

- Prepublication history and additional material for this paper is available online. To view these files please visit the journal online (http://dx.doi.org/10.1136/ bmjopen-2013-004176).

Received 4 October 2013 Accepted 14 October 2013

CrossMark

Faculty of Nursing, Edmonton Clinic Health Academy, University of Alberta, Edmonton, Alberta, Canada

Correspondence to Rubina I Barolia; barolia@ualberta.ca

\section{ABSTRACT}

Introduction: There is a misconception that cardiovascular disease (CVD) is the burden of wealthy nations, but, in fact, it is the leading cause of death and disability-adjusted life worldwide. Healthy diets are an essential factor in the prevention of CVD. However, promoting healthy diet is challenging, particularly for people with low-socioeconomic status (SES), because poverty is linked with many risk behaviours such as smoking, unhealthy eating and obesity. Multiple factors, cultural values and beliefs interact and make healthy eating very challenging. The effects of these factors in the context of low-SES populations with CVD are largely unknown. To address this gap, this study will examine the factors that affect decisions about consuming healthy diet in Pakistanis with low SES who suffer from CVD.

Methods and analysis: A qualitative method of interpretive description will be used. 25 participants will be selected from two cardiac rehabilitation (CR) centres in Karachi, Pakistan. Face-to-face interviews using a critical realist framework will be used to understand individual and contextual factors in the food choices of people with low SES and CVD. ATLAS.ti qualitative data analysis software will be used to identify themes and patterns in the interview data.

Ethics and discussion: Ethical approvals were received from the Ethics Review board of University of Alberta, Canada and Aga Khan University, Karachi Pakistan. The findings will generate new knowledge about which and how factors influence the food choices of Pakistanis with CVD and low SES to provide an insight into the development of an operational framework for designing interventions for prevention of CVD. For knowledge-translation purposes, we will publish the findings in highly accessed, peer-reviewed scientific and health policy journals at the national and international level. This research protocol received IRDC (International Development Research Centre) doctoral award from International Development Research Centre, Ottawa, Canada.

\section{Strengths and limitations of this study}

- The study will identify the reasons that primary and secondary prevention guidelines and teachings are not effective in changing health-related behaviours, particularly for patients with CVD with low income.

- The findings from this study will generate new knowledge about the factors that influence food choice in low-income groups of Pakistanis so that it can be incorporated into primary and secondary preventive programmes for CVD in the Pakistani context.

- People who live below the poverty line in two settings in Pakistan will be interviewed; therefore, the findings cannot be extrapolated to all income groups of people of Pakistan.

\section{INTRODUCTION}

There is a misconception that cardiovascular disease (CVD) is the problem of only wealthy nations. In fact, it is the leading cause of death and disability-adjusted life worldwide. Recent evidence has shown that more than $30 \%$ of deaths are due to CVD and that about $80-86 \%$ of these deaths occur in lowincome and middle-income countries (LMICs). ${ }^{1-3}$ There is, however, some variation in mortality rates according to age, gender, socioeconomic status (SES), ethnicity and geographical location. The CVD death rate is three times higher for men at a younger age (less than 70 years) than for women and twice as high in socioeconomically disadvantaged areas than in more affluent areas. ${ }^{4}$

In the past decades, CVD death rates have declined in several developed countries but increased in LMICs. According to the Global Burden of Disease Study, the disabilityadjusted life years loss (a measure of overall 
disease burden, expressed as the number of years lost due to ill health, disability or early death) due to CVD will rise 55\% between 1990 and 2020 in developing countries. In contrast, the mortality rates due to CVDs will decrease $14.3 \%$ in developed countries.$^{5}$ The South Asian countries of Pakistan, India, Bangladesh, Sri Lanka and Nepal constitute more than a quarter of the developing world and are known to have a higher coronary risk than any other part of the world. ${ }^{6} 7$

Healthy diet is one of the most essential factors in the prevention of CVD. Consuming healthy diet is considered more beneficial than taking medication for longer periods of time to prevent chronic diseases such as CVD. ${ }^{8}$ Scientific literature has shown that dietary patterns are closely related to cultural values, beliefs and various economic factors. ${ }^{9}$ Multiple factors interact to make healthy eating very challenging and sometimes impossible. The effects of these factors on the food choices of poor people who have CVD are largely unknown.

To address this gap in the research, in this qualitative study we will focus on understanding the factors that influence the food choices of Pakistanis with low income and CVD. We will also identify the influences of sex/gender on the participants' food choices. This research is guided by the theory of critical realism, which is widely used to examine complex phenomena that require a deeper level of understanding. The study findings will generate new knowledge on the factors that influence the food choices of groups of people of low-SES to incorporate it into secondary preventative programmes for CVD.

\section{Definition of terms}

A healthy diet normally implies an increased level of relative consumption of fruits, vegetables, whole grain, low-fat milk, fish and olive oil or vegetable oils for cooking and a reduced intake of red meat, saturated fats, hydrogenated fat and salt. ${ }^{10-12}$ This pattern of eating can delay the development of atherosclerosis, thus reducing the incidence of coronary events and being an important modifiable risk factor in the prevention of secondary cardiac events. ${ }^{13}$

Healthy eating is a broad concept with diverse interpretations found in the literature in terms of culture, behaviour and health-related issues. ${ }^{14}$ In this research healthy eating is used in terms of consuming healthy diets that are recommended for patients with CVD. We have used the terms healthy diet and healthy eating synonymously, unless otherwise indicated.

Food choice in this research refers to the food decisions or food selections of patients with CVD. Food selection does not have to be based solely on people's health and nutrition beliefs, but involves financial, social, lifestyle, environmental and family issues. ${ }^{15}$ It is important to understand how patients with CVD make these decisions.

Sex is defined 'in terms of biological difference, whereas gender refers to economic, social and cultural attributes. $^{, 16}$
CVD refers to a disease caused by a series of pathological processes in which the coronary arteries gradually thicken and harden, leading to atherosclerotic plaques that impede or occlude coronary and other vascular blood flow. This leads to such clinical manifestations as angina and acute coronary events such as acute coronary syndromes, myocardial infarction, heart failure and other peripheral vascular diseases that result in disability and death. ${ }^{2} 1718$

\section{Knowledge gap and current evidence Heath transition and CVD risk}

In the past decades, the health and illness profiles of people have changed markedly. Yusuf $e t a l^{3}$ reported that industrialisation, urbanisation, and the aging population have resulted in a double burden of diseases, particularly in LMICs. These countries are already facing a high prevalence of infectious, nutritional and perinatal diseases; the forces of globalisation, urbanisation and industrialisation have changed people's lifestyles, led to high consumption of energy-rich foods and decreased energy expenditure through decreased physical activity. ${ }^{4}$ The lack of equity in the healthcare systems of many countries has widened the gap between the rich and poor. Therefore, people of low-SES do not have access to healthcare facilities in high-income, and LMICs. Clark $e t a l^{19}$ critically examined the association between SES and CVD and found that 'health is not confined to high-income countries nor to the postindustrial age; ... substantial health inequalities exist in both within and between countries' (p.712). Low SES is therefore one of the independent risk factors for or the predictor of the development of CVD in men and women. ${ }^{19}$

Moreover, the increasing life expectancy, economic instability, poverty and inequitable health facilities are also accelerating the rates of CVD in LMICs, including Pakistan. Increasing longevity has influenced the lifestyle and the exposure of people to the risk of CVD for a longer period of time. Thus there is a high chance that CVD events will manifest at a later age. ${ }^{5}$ The most degenerative changes in blood vessels and vital organs, such as diabetes and hypertension, also start at a later age, which also increases the prevalence of CVD. ${ }^{20}$

\section{Preventive guidelines are not effective in changing health-related behaviours}

Health-related behaviours such as smoking, diet and physical activity have a major impact on morbidity and mortality related to CVD and many other chronic diseases. Healthy populations are more productive, enjoy a better quality of life and place less of a burden on the healthcare and social systems. ${ }^{14}$ The American Heart Association's and European Society of Cardiology's dietary guidelines for CVD prevention recommend high intake of fruits, vegetables, whole grains, skim milk and fish and a reduced intake of red meat, saturated fats, hydrogenated fat and salt. ${ }^{11}{ }^{12}$ In addition to these guidelines, messages on healthy eating for public 
awareness have become widespread, delivered through mass media at multiple levels of society. The Pictorial Canada food guide, healthy eating booklet, food pyramid pamphlet and awareness programmes are some of the examples of health messages that are conveyed to people to promote healthy eating.

Despite these clear and comprehensive guidelines, in reality, their practical application is not straightforward. There is a huge gap between healthy diet messages and people's eating practices. ${ }^{9} 1421$ Because an individual's lifestyle is shaped by numerous factors, including family and sociocultural background, environment, religion, education and personal knowledge, changing one's lifestyle or behaviour can potentially be extremely difficult. $^{14}$

CR programmes have proven beneficial for the secondary prevention of CVD; however, these programmes do not achieve lifestyle changes such as cessation of smoking, better eating habits and increased physical activity. For example, a European CR inventory survey showed that less than $50 \%$ of people benefit from CR programmes. $^{8}$

Similarly, European Action on Secondary and Primary Prevention by Intervention to Reduce Events in nine European countries ${ }^{22}$ showed that the trend in smoking, obesity and central obesity increased between surveys 1 and 2 because of an increased trend in risky lifestyles; therefore, Kotseva et $a l^{22}$ recommended

raising the standards of preventive cardiology through more effective lifestyle intervention, control of other risk factors and appropriate use of cardio protective medication. It is possible to achieve a higher standard of preventive care for coronary patients and their families with the use of professional, comprehensive and multidisciplinary team approaches. (p.125)

There may be other reasons for ineffective CR programmes, such as non-referral of eligible patients, geographical location and poor patient motivation. Women, older adults and ethnic-minority patients are utilising this facility even less. ${ }^{23}$ Therefore, it is very important to assess and analyse the factors that promote and hinder changes in lifestyle at the societal, institutional, professional and patient levels, which are likely to be different in different parts of the world.

\section{Increasing CVD in South Asian countries}

This increase is evident in many studies conducted in Pakistan and other South Asian countries. For example, Dennis $e t a l^{24}$ studied a mixed population in Karachi, Pakistan, and found that $16 \%$ of men and $24 \%$ of women had high cholesterol and that $34 \%$ of men and $49 \%$ of women were obese. Similarly, Joshi et $a l^{25}$ reported that the South Asian population has high lipid levels $(48 \%)$, a high rate of obesity (waist to hip ratio of $37.7 \%)$ and other risk factors at a younger age $(<40$ years) than that of the people of other countries.
Studies conducted with immigrant populations of developing countries also identified a high risk for developing CVD. ${ }^{25}$ Kumar et $a l^{26}$ estimated a 10-year risk of cardiovascular death between ethnic Norwegians and five immigrant groups (from Turkey, Iran, Pakistan, Sri Lanka and Vietnam) and found that all immigrant groups have a high estimated risk of cardiovascular death except for those from Vietnam. The risk varies from $7.7 \%$ (Turkey/Pakistan) to $1.5 \%$ (Vietnam). ${ }^{26}$

The assessment of cardiovascular risk from an individual country's perspective is a key factor in prevention. Therefore, a large-scale INTERHEART global case control study was conducted in 52 countries throughout Asia, Europe, the Middle East Crescent, Africa, Australia and North and South America. This standardised study identified nine modifiable risk factors for CVD: current smoking, diet, diabetes, hypertension, obesity, stress, exercise, alcohol and high blood lipid levels (ApoB/ ApoA1rati). These risk factors accounted for a very high population-attributable risk in every ethnic group (Europeans, 86\%; Chinese, 90\%; South Asians, 92\%; Black Africans, 92\%; Arabs 93\%; and Latin Americans, $90 \%)$. However, the age of presentation varies in different regions, with the youngest patients in South Asia and the Middle East (46-61 years) and the oldest patients in Western Europe, China and Hong Kong (54-74 years). ${ }^{3}$ The younger age of heart disease patients in South Asia is due to the high prevalence of all risk factors at younger ages. $^{25}$ The INTERHEART study results show that these risk factors are consistent in men and women across different geographic regions and by ethnic group, which makes the study applicable worldwide.

The findings from the individual studies conducted on CVD risk factors and the INTERHEART study suggest that the major emphasis of future research should be on understanding why and how these known risk factors can be reduced to identify population-specific approaches and prevent CVD. ${ }^{3} 1021$

\section{Factors that influence lifestyle and dietary change for people with CVD}

Health-related behaviours such as smoking, diet and lack of physical activity have a major impact on morbidity and mortality related to CVDs and many other chronic diseases. An individual's lifestyle is shaped by numerous factors, including family background, sociocultural environment, religion, education and personal knowledge. Some of the most important factors that impact CVD mortality and morbidly, particularly for people of low-SES, include the following.

\section{Economic and educational factors}

It is generally accepted that better health requires money with respect to a healthy diet, a living environment that is conducive to good health and access to healthcare. Poverty and ill health have a complex relationship. Better health reduces poverty, and reduced poverty improves health. $^{27}$ In a qualitative study 
conducted with low SES patients in Canada, Clark et $a l^{10}$ also identified multiple factors that influence the food choices of people with CVD. They found that although the participants were knowledgeable about the principles of a healthy diet, they expressed the realities of life in the context of economic constraints and their inability to buy healthy foods. They felt powerless and guilty because of their lack of compliance with a healthy dietary regime and more stressed because they were unable to meet other necessities such as house rent, bills, transportation and medications. ${ }^{10}$ The results of the INTERHEART study also show that the $50 \%$ increase in myocardial infarction associated with low education is a result of lifestyle factors. ${ }^{28}$ Therefore, in circumstances of resource constraints, people are often unable to spend money on healthy food and education, although in many instances poverty-related stress has led people to spend money on smoking and alcohol rather than on healthy food and education.

\section{Gender influence}

Given the influence of gender and culture, it is likely that much remains to be learned about dietary patterns, eating habits and food choices. The few studies that have been carried out to understand the dietary habits of patients with CVD noted that a high proportion of patients is unable to change their dietary habits. ${ }^{29} 30$ Some of the reasons for not following a healthy diet according to published studies include "social pressure during eating, gender role, taste, cost, food preparation, lack of self-control and family support." ${ }^{\text {"3 }}$ These studies have not examined the barriers that prevent a change in diet for people of low income who have CVD.

Gender influence in every culture has also impacted dietary choices of household members. In many South Asian cultures, food is cooked in accordance with men's preferences. The clinical manifestations of heart disease in women are found to be more intense and fatal than those in men. ${ }^{31}$ The onset of the disease also occurs at a later age for women, at which there is a greater risk of CVD due to age-related comorbidity. ${ }^{16}$ Therefore, assuming that men and women have similar disease-risk profiles could be misleading.

\section{Sociocultural and religious factors}

Sociocultural factors, including values, beliefs, education, religion and environment, influence the way that we live, make health-related decisions, and form our perceptions of health-related activities. Lifestyles, particularly with regard to eating habits and smoking, are culturally and ethnically determined. Society influences lifestyle by shaping the accepted norms and defining social controls. For example, smoking is a socially acceptable norm for men in some South Asian cultures. In particular, in the Bangladeshi community it represents a "strong sense of social bonding and normal part of being men." ${ }^{22}$ In some cultures, overeating during adolescence and pregnancy is encouraged to the extent that it can lead to obesity. ${ }^{1}$

\section{Structural factors and political influences}

Globalisation, trade and food marketing have also had a negative impact on people's lifestyle. For example, the political nature of food production and distribution is such that a small number of transnational food corporations control food production and retailing, which makes healthy food expensive. Increasingly ubiquitous fast-food chains have also affected people's dietary habits. Because supermarket foods tend to be highly processed and have long shelf lives, people buy them for economical and practical reasons (such as time). Food marketing (fast food) and advertising companies (in related fields) also play an important role in changing people's lifestyle. ${ }^{33}$

According to Appel et $a l,{ }^{34}$ various types of behaviourintervention trials have also been assessed to determine the effects of intervention on lifestyle change. These interventions are based on behaviour-modification theories and models that have been proposed to explain behaviour change. Appel et al criticised the positive results of these interventions because these trials usually "enrolled motivated individuals" ( $p .303$ ) who were more likely to be ready for change. In addition, these studies did not assess the changes over a long period of time (usually for only 6-8 months). Problems related to complex structures and political forces arise when individuals are unable to change their lifestyle.

An in-depth understanding of culture, values, beliefs and health-related behaviours in relation to the above risk factors in the context of individual countries is necessary to develop culturally appropriate riskmodification strategies in these countries. Understanding the social and economic issues and local context, resources and capacities of specific settings is also very crucial to design contextually based preventive interventions to reduce the CVD risk factors, particularly in LMICs. The burden of these risks factors can be reduced by modifying lifestyles; otherwise, the increased risk factors are expected to double in the next decades. $^{35}$

\section{Research objectives}

1. Understand the factors that support and inhibit the consumption of healthy food choices of Pakistani people of low-SES who have CVD.

2. Identify the influence of gender/sex in the food choices of people of low-SES who have CVD.

3. Explore pertinent issues with regard to food choices and the dietary practices of people of low-SES who have CVD.

\section{Research questions}

1. What factors inform the decisions of Pakistani people of low-SES on food choices after a diagnosis of CVD? 
2. What factors promote the consumption of healthy diets of people of low-SES who have CVD?

3. What factors inhibit the consumption of healthy diets of people of low-SES who have CVD?

4. How does gender/sex influence the dietary practices and food choices of people of low income who have CVD?

\section{Context of the study}

The Islamic Republic of Pakistan, located in southern Asia, consists of $852382 \mathrm{~km}^{2}$ and shares borders with India, China, Iran, Afghanistan and the Arabian Sea. ${ }^{36}$ Pakistan is the sixth most populous country, with a population of 175 million in its four provinces. Sixty-four per cent of the people live in rural settings and have limited access to health resources. The per capita gross national income of Pakistan is US\$470, which is much lower than that of developed countries such as Canada (\$C20 510). One-third of Pakistan's people live below the official poverty line (less than US $\$ 1-U S \$ 1.25$ day, or less than ₹98/day). ${ }^{37-40}$ Five major ethnic groups-Punjabis, Sindhis, Baluchis, Pakhtuns and Muhajirs-originate from various parts of Central and South Asia. Each group has its own distinct language, place of origin, cultural practices, dietary habits, health beliefs and behaviours. More than 20 dialects are spoken throughout the four provinces of Pakistan. ${ }^{37}$ The ethnic variation and different dialects in Pakistan have also made the distribution of the CVD risk factors complex and multifaceted. The major issues include poor governance and inappropriate resource allocation; eg, more resources are allocated to tertiary care than to primary and secondary prevention. ${ }^{41-43}$ Considering the phenomenon under investigation in this study about promoting healthy diets in Pakistan, it is worthwhile discussing Pakistan's economic status, the CVD risk profile, the healthcare system of the country and gender issues.

\section{Potential use of the research}

This research will generate substantive and contextbased data to guide the development of preventive guidelines for the cardiac rehabilitation (CR) centres in hospitals and community settings. The findings might also be used to develop an operational framework for healthy diets to prevent CVD in low-income settings in Pakistan. The study findings can be used at three levels:

1. Individual and family level: Healthcare providers will be able to set realistic goals for low-income patients that will not focus solely on education with regard to eating a list of food items that are prescribed in the current recommended guidelines. Educational strategies can improve patients' knowledge of their lifestyle and disease management if they address the cultural values and available resources to purchase food. For example, people cannot follow dietary advice if it is not culturally acceptable. However, if the dietary advice conforms with their own dietary patterns and food combination, they might willingly consider the change. Similarly, local food items that are cheaper and have similar nutritional value can be suggested as options.

2. Health system and structural levels: The findings from this study can inform healthcare organisations about preventive measures that require broader programmatic and structural changes for the public or private sectors that provide healthcare to poor people.

3. National policy level: CVD prevention cannot be achieved in isolation; therefore, health policies at the national and regional level should be integrated into other systems such as finance, education, and the social and environmental structures. This study can play a pivotal role in formulating recommendations for government and non-government organisations to prevent fragmented services and might call for major changes in health and agricultural policies and resource allocation as well as practical improvement in the environment for people of low income.

\section{DESIGN/METHODS}

Studies have shown that healthy diet reduces the development of CVD worldwide; $;^{4} 10134445$ it is, therefore, important to understand why some people change their diet, whereas others do not, particularly in the context of low-SES. A qualitative examination with an interpretive descriptive approach can clearly address such a question. Interpretive description (ID) answers complex, experiential and contextually based research questions that are relevant to nursing and other healthcare disciplines, whereas existing traditional qualitative approaches cannot. ${ }^{46}$ Interpretive descriptive methodology has a practical goal and fosters an understanding of the phenomenon on the basis of available empirical evidence. ${ }^{46}$

According to Thorne, ${ }^{46}$ these methods developed from the social sciences are firmly grounded in their own theoretical and empirical assumptions and might not address the practical problems of nursing. In certain cases, researchers who follow these conventional approaches are also expected to follow their methodological rules to ensure the quality of their studies. Therefore, the results of such studies can be "incongruent with their stated methodological choices and the research product is a mixture of different methodologies without convincing justification. ${ }^{47}$

Philosophically, ID orients itself with an interpretive naturalistic perspective, which constructs reality in the course of interactions between the researcher and the participants in a natural environment. ${ }^{48}$ Reality in a naturalistic paradigm cannot be understood in isolation; rather, it must be constructed in the context of complex human experience. ${ }^{49}$

Thorne ${ }^{46}$ considered key axioms from the naturalistic paradigm, because inquiry into a disciplinary problem has several common sets of assumptions about reality. The key axioms of the ID include the following: (A) the lives of the 
participants are a construct of multiple realities that are "complex, contextual, constructed and ultimately subjective", ${ }^{\circ}$ (B) the cultural, social and linguistic contexts in which each person lives continually shape and reshape his or her realities; $(\mathrm{C})$ the researcher and participants come together to create their understanding of the phenomenon being studied; (D) the focus is on understanding human commonalities; however, variant individual perspectives are also honoured and valued and (E) the researcher does not take a position of objective distance, situating himself or herself apart from the participants, but engages with them in their naturalistic world to maintain respect and ensure their comfort and ethical rights. ${ }^{49}$ Congruency among these assumptions results in legitimate knowledge generation.

ID often involves multiple data-collection strategies such as interviews, participant observations and documentary analysis to make comprehensive and contextualised interpretations of the central phenomenon of interest. ${ }^{46}$ In general, the samples are purposively and often theoretically generated. Theoretical sampling is an approach in which the participants are recruited based on the need for information and the need to make 'varied analytical and relevant distinctions' rather than to determine 'the frequency of the occurrence. ${ }^{51}$ Thorne $^{46}$ did not support the notion of data saturation because of the infinite variation in participants' experiences in the disciplinary context of health research. When the data are of sufficient depth to meaningfully describe the phenomenon under study, the researcher has reached the concluding point for the data collection. ${ }^{46}$

Researchers use an inductive process to understand patterns and structures of the phenomena under study ${ }^{50}$ Concurrent data collection and analysis and a constant comparative method in an iterative manner are recommended to verify the findings. ID focuses on broad themes and the overall picture of the phenomena as a coherent description of common themes and patterns, taking into account individual variation. The study results also reflect clinical practice that makes sense to the practitioner rather than listing off free-floating, isolated themes. ${ }^{46} 50$ Thorne $^{46}$ did not support the notion of data saturation because of the infinite variation in participants' experiences in the disciplinary context of health research. When the data are of sufficient depth to meaningfully describe the phenomenon under study, the researcher has reached the concluding point for the data collection. ${ }^{46}$

The findings of such studies create a platform for assessment, planning and interventional strategies that can be logically and ethically applied in clinical settings. In short, ID leads to the careful and systematic analysis of a phenomenon and simultaneously allows the researcher to use that analysis in the context of the practice field.

\section{Theoretical framework}

The main tenets of the theory of critical realism will be used to guide this study. Critical realism accounts for individual factors and structural factors that influence dietary decision-making. ${ }^{52}$ Individual factors include participants' knowledge, beliefs and attitudes; whereas structural factors include gender, culture, social norms, and the political and geographical environments. ${ }^{10} \quad 52$ Critical realism is derived mainly from the philosophy of Bhaskar, ${ }^{53}$ who argued that the purpose of science is to describe social phenomena in a way that makes sense to us and not just to seek the absolute truth as in experiments. ${ }^{52-54}$ Critical realism stratifies reality and suggests that reality is influenced by various elements, objects, practices and structures. It has three domains: “(1) the empirical-which are experienced and perceived, (2) the actual-actions that can be observed, and (3) the real-underlying structures, powers and tendencies which cause events in an actual domain." ${ }^{52}$ These domains identify the underlying structures, powers or forces that influence people's behaviour or way of living, even though they might not be aware of them. Thus, critical realism focuses on explaining the outcomes as they occur in complex and natural environments.

The theory also helps us to understand the broader social structures such as poverty, sex, race and so on that exist in society and influence behaviour. ${ }^{52}$ People cannot deny the existence of these social realities just because they do not recognise or perceive them as factors that affect their behaviours or actively influence their lifestyle patterns..$^{55}$ The way that people live can be understood in complex natural settings in which multiple factors act together to form behaviour. Some of these factors are physical, social, cultural, economical, environmental and geographical. ${ }^{55}$ In summary, critical realism offers a comprehensive approach that explains the outcomes in real and complex environments. It explores the influences of the underlying powers and structures that the participants might not have perceived as barriers in selecting a healthy diet.

\section{Study setting}

This study will take place in Karachi, the largest metropolitan city of Pakistan, which has a population of approximately $18-20$ million. ${ }^{56}$ Many ethnolinguistic groups from all parts of Pakistan reside in Karachi, and most of the people in Karachi are of low-income and middle-income groups. The main causes of death for adults are CVD, injuries and complications from pregnancy. ${ }^{57}$ We aim to recruit participants mainly from CR centres or any other cardiac facilities of the two large hospitals in Karachi, where people with low-SES receive rehabilitative services free of charge. One rehabilitation centre is at Aga Khan University Hospital (AKUH), and the other centre is located at the National Institute of Cardiovascular Diseases (NICVD). Both centres provide 2-4 weeks of comprehensive services, which include education, prescribed exercise, nutritional counselling and other behavioural interventions. ${ }^{58}$

NICVD was established in 1963 to meet the national need to cope with the increasing demand for diagnosis, 
management and prevention of CVDs. The institution provides diagnostic, preventive care and medical services to all patients free of charge. Most people from low-SES from all parts of Pakistan are referred to NICVD for the diagnosis and treatment of CVDs. The daily out-patient attendance averages 1200 individuals, and an average of $30-40$ patients attend rehabilitation services. ${ }^{59} 60$

AKUH started its operation in 1985. It is a not-for-profit, private teaching institution committed to providing the best possible options for the diagnosis and management of all diseases for all patients. Although AKUH is a private institution, it provides financial assistance to needy and poor patients who cannot bear the cost of medical treatment and rehabilitative services. AKUH's Department of Patients' Welfare is responsible for screening patients' needs and providing financial assistance accordingly. ${ }^{61}$

\section{Study sample}

We anticipate that 25-30 participants, 12-15 from each facility, will be sufficient to provide us with meaningful descriptions to answer the above research questions. Hunt $^{47}$ used 18 participants to investigate the 'moral experience of Canadian healthcare professionals in humanitarian work' and achieved sufficient depth and meaningful description. Hunt further explained that purposive sampling results in a diverse set of participants, and theoretical sampling achieves richness in understanding the issues "identified in the early analysis of the data, or who could address aspects of the inquiry that remained underdeveloped or thin. ${ }^{, 7}$ We will recruit participants who have financial assistance at any cardiac facility of AKUH and include participants from NICVD if they earn \$C1.25 (Rs.113) or less, based on the poverty line defined by Pakistan's economic analysis. ${ }^{62}$ We will purposively and theoretically select participants to capture expected and emerging data variations. Purposive sampling will include participants who represent different age groups, men and women, different ethnicities, those with different educational and income levels and those who are willing to share their decisionmaking process on food choices; whereas theoretical sampling will involve participants based on information that emerges from an early data analysis. Recruitment of patients from the private and public healthcare settings will also yield maximum variation in sampling.

\section{Inclusion criteria}

The participants in this study will include adult patients with CVD who (A) are aged 30-70 years with a documented CVD diagnosis. As mentioned earlier, the onset of CVD in South Asian population occurs at a younger age. People younger than 40 years have also developed heart disease because of the high prevalence of all risk factors at a younger age ${ }^{25}$; (B) have attended any kind of CR programme or been taught secondary prevention; (C) earn \$C1.25 (Rs113) or less per day based on the poverty line defined by Pakistan's economic analysis (self-reported); (D) are of any ethnic group or religion; (E) are able to speak English or Urdu (the national language of Pakistan) and (F) are willing to talk about and reflect on their experiences with the phenomenon under study.

\section{Recruitment process}

We will recruit participants by word of mouth and briefly explain (in Urdu) this study during the rehabilitation sessions. We will also request that the staffs who work in the clinics identify eligible participants and obtain their contact numbers or email addresses. The primary author will contact the potential participants by telephone or email. During the initial conversation or meeting she will ensure that each individual meets the inclusion criteria and will arrange times and places for the interviews that are suitable to the participants. Otherwise, she will interview them in one of the offices that AKUH or NICVD will make available to her.

\section{Data collection}

\section{Literature and document review}

A critical review of peer-reviewed and non-peer-reviewed literature on the influences of economic status on food choices in Pakistan, which we mentioned earlier in the background and recent evidence section of this protocol, will form the preliminary analytic framework. However, during the data-collection process, we will also conduct an ongoing literature search to explore the emerging factors that promote or prevent healthy eating among patients with CVD of low-income groups. The literature will also include national economic reviews, policy documents and surveys completed in the Pakistani context.

\section{Participants' interviews}

With ID, themes and patterns can be extracted by studying the subjective perceptions of the research participants, thus creating a real understanding of the phenomenon of interest. ${ }^{63}$ At the participants' preferred locations the primary author will conduct a minimum of two face-to-face, semistructured, individual interviews in Urdu, in which she is fluent, to identify the facilitators of and barriers to healthy eating. At the first meeting, prior to conducting the in-depth interviews, she will read and give the potential participants a letter of information about the study in Urdu or English (see online supplementary appendix 1) and obtain their informed consent to participate (see online supplementary appendix 2). She will then request that each participant complete a demographic information sheet (see online supplementary appendix 3). In addition, she will reassure the participants that she will maintain their right to confidentiality and privacy during the interview and data-analysis process and establish trusting relationships. In the second interview she will obtain rich, in-depth information by using the interview guide (see online supplementary appendix 4 ). 


\section{Interview guide}

The interview questions will be guided by the theory of critical realism ${ }^{52} 64$ to identify the contextual and individual factors that determine the participants' food choices and issues related to healthy eating. The interviews will be composed of open-ended questions aimed at eliciting the participants' knowledge of healthy diets, who decides to purchase food, how they make these decisions, how successful they are in changing their diets and the difficulties that they face in selecting food (see online supplementary appendix 4 ). The guide will cover the educational, financial, cultural, structural and gender influences. It will be pilot-tested on two participants before the interviews begin to ensure the participants' clarity on and understanding of language and concepts. After the interviews are piloted, we might modify the interview guide to gain more in-depth information. We will include the pilot interviews in the data analysis.

\section{Recording data}

For analytical and reporting purposes, one of the bilingual research assistants from the AKUH research office will translate all of the transcripts into English. Although this individual has expertise in translation, the primary author will also verify the accuracy of the translations. Each interview will likely last $45-75 \mathrm{~min}$, and the interviewer will audiotape it with the participant's permission. Immediately after each interview she will write and maintain field notes to reflect the participants' non-verbal behaviours and emotions. A reflexive journal will be maintained to record the overall process of data collection. The data collection will conclude when sufficient and meaningful depths have been achieved.

\section{Data analysis}

The data collection and analysis will occur concurrently. Soon after each interview, the primary author will transcribe the recording verbatim and then code the transcript to reveal broad or initial categories or themes. She will also maintain field notes immediately after each meeting that will include her reflections on the interview process, the participants' non-verbal behaviours and the initial themes that emerge from the interviews. The transcripts will then be analysed and discussed with the research team, and the data will be examined for similarities and differences in patterns to understand and gain insight into the phenomenon in the participants' context.

To manage the participants' data, we will use ATLAS.ti Scientific Development Software, which facilitates the organisation of the data and arrangement of the datasets at different levels of the analytic scheme. ${ }^{65}$ We will also critically examine the data for underlying structures that the participants might not perceive as barriers to healthy eating.

The final analysis will thus convey the entire essence of individual and contextual factors that influence the participants' dietary decision-making. We will discuss the themes and categories in the final stage with the research team members, who are subject-expert health professionals. Verification and discussion of the themes with the experts will also ensure that the analysis of the data reflects the participants' views, makes sense and will be useful in the practice setting.

Thorne $^{46}$ suggested that the findings should create a platform for assessment, planning and interventional strategies that can be logically and ethically applied in clinical settings. ${ }^{46}$ We anticipate that this study's findings will lead to the careful and systematic analysis of the contextual and individual factors that influence the participants' healthy eating and will simultaneously allow me to use that analysis in assessment, planning and interventional strategies in the practice field. Table 1 summarises the analytical steps.

\section{Strategies to ensure the rigour of the study}

To ensure the quality and rigour of the study, we the researchers will be deeply involved with the data (eg, transcribing, reading and rereading the transcripts; conducting an inductive analysis) and will maintain transparency while subjectively analysing the data. We are aware of social structures, power relations and gender influences in Pakistan and will, therefore, explicitly acknowledge any personal biases that could influence the research findings.

Thorne $^{46}$ suggested some guiding principles to ensure the quality of the findings that result from the use of the ID method, and we will apply these principles throughout the entire research process. These principles are as follows:

1. Epistemological integrity is achieved by ensuring the logical flow of the research questions, data sources and data interpretation.

2. Variant sampling will include a broad age range, males and females, diverse ethnic backgrounds and samples from two different rehabilitation centres to help us to gain substantive knowledge of the phenomenon that cannot be produced simply by selecting similar patients from similar backgrounds. Prolonged engagement with the data and a review of prior literature can result in representative credibility and substantive completeness.

3. We will use Miles and Huberman's ${ }^{66}$ approach for data analysis, which requires an explicit description of the researcher's analytical thinking. We will describe the decision-making process while developing the initial and final themes. This also allows novice researchers to learn the practical application of analytic structures and assists in replicating the research process should other researchers want to repeat it. We will remain vigilant throughout the analytic process and will not attempt premature closure of our early conceptualisations. This will help us to articulate and contextualise specific analytic decisions within the larger picture to reflect analytic logic. 
Table 1 Analytical steps

\begin{tabular}{|c|c|}
\hline Activity & Expected analysis \\
\hline Listening to the interviews & Being immersed in and becoming familiar with the data \\
\hline Relistening to the recordings & Making sense of the data \\
\hline Reading reflective notes and transcripts & Getting an overall picture of the data \\
\hline \multirow[t]{6}{*}{$\begin{array}{l}\text { Mapping the coding scheme (using Atas.ti qualitative } \\
\text { analysis software) }\end{array}$} & $\begin{array}{l}\text { Questioning What is happening here? What am I learning } \\
\text { about this? and Why is this here? }\end{array}$ \\
\hline & Identifying codes and categories and linkages \\
\hline & Grouping similar categories \\
\hline & Forming themes and patterns \\
\hline & $\begin{array}{l}\text { Examining the similarities and differences in themes and } \\
\text { patterns }\end{array}$ \\
\hline & $\begin{array}{l}\text { Exploring relationships and patterns among the data sources; } \\
\text { concept mapping may be useful }{ }^{59}\end{array}$ \\
\hline Validating analysis & Consulting the members (experts) \\
\hline Clarifying issues (if required) & Going back to the participants \\
\hline Additional interview (if required) & $\begin{array}{l}\text { Conducting more interviews with the same or other participants } \\
\text { Consulting the literature regarding the themes and patterns }\end{array}$ \\
\hline $\begin{array}{l}\text { Moving in and out of the data and following the above steps } \\
\text { in an ongoing, nonlinear (circular) fashion }\end{array}$ & $\begin{array}{l}\text { Identifying a range of alternatives to gain the essence of the } \\
\text { phenomenon under study for the final analysis }\end{array}$ \\
\hline
\end{tabular}

4. Thorne ${ }^{46}$ defined interpretive authority as the transparent presentation in the research report of the power relation, the subjective construction of knowledge and the researcher's position in the study. This principle emphasises the researcher's trustworthiness in interpreting the data. ${ }^{46}$ Therefore, as researchers, we will explicitly identify any personal biases and power relations and take ownership of the interpretation and findings from the data.

Synonymous with the goals of the paradigm, ID does not aim for generalisability of the findings; however, the commonality of the patterns that we identify in the study might help to generalise the findings within the context and the time. ${ }^{46}$ This study will foster a deeper understanding of the complexities of the health-promoting eating behaviours of poor people with CVD. The knowledge gained from this study will provide a more complete picture of its ground realities, facilitate the design of comprehensive preventive interventions for CVD in the context of low-SES and inform future research and national health policy development for required programmatic and structural changes to promote healthy eating. Communities will also be able to draw on the study's findings to make the necessary changes in their own environments to facilitate changes in their shared eating habits. In Pakistan, tertiary care for the management of CVD receives a great deal of emphasis, but it is very costly for the poor. The promotion of primary and secondary preventive programmes is critical for the country to overcome the burden of CVD in a costeffective manner.

\section{Information letter and consent}

The author submitted an information letter to satisfy the informed-consent standards and a separate permission letter to appropriate management teams at the selected field sites. The letter is also written in Urdu for participants who do not understand English. When necessary, we will offer information about the study and the consent procedures verbally in Urdu. The information letter includes details on voluntary participation in the study, the potential benefits and potential harm from participation in the study and the right to withdraw from the study at any time without penalty. We will maintain the participants' anonymity and confidentially throughout the research process and during the dissemination and publication of the findings. We will ask the participants to sign or place a thumb impression on the consent form.

We will keep the raw data and all identifying information in a separate locked cabinet and the soft copies in encrypted computers. Other than the research team, no one will have access to the participants' data. When we present the study's findings, we will use pseudonyms to protect the participants' identities. We will also maintain their anonymity during the tape recording and transcribing, translating and analysing of the data. We will keep the data for 5 years as per the University of Alberta's policy and will electronically erase the data from the audiotapes and shred the hard copies of the transcripts and analysis in a confidential shredder.

\section{Monetary compensation}

At the end of the first interview, we will give each participant Rs.250 (approximately \$C2.5) as a token of thanks for their participation. We will not ask any participants who wish to withdraw from the second interview to return the money. 


\section{Gender implications}

As we discussed earlier, Pakistan is a patriarchal society in which women are primarily responsible for all household chores and men are considered breadwinners responsible for the household earnings. With limited resources, the female family members usually select and prepare the food. It is also customary that family practices determine the food to be eaten and that all family members eat the same food prepared in the house. Thus, acceptable cultural norms and resource constraints limit separate food preparation and food selection for patients with CVD. Because the onset of CVD in women tends to occur at a later age when there are more age-related comorbid issues, the management of CVDs and secondary prevention becomes more challenging and complex. ${ }^{16}$ The findings of this study will categorise the difficulties that strict gender roles present for healthy eating and identify gender-specific approaches for prevention and counselling. The study results will also identify the resource constraints that female family members face in making food choices for their families, as well as identify the limitations of any family.

\section{Communication of findings and knowledge transfer plans}

A multilevel knowledge translation plan is created to ensure that key messages are developed for specific audiences. ${ }^{67}$ The principal audience for this project is the rehabilitation centre staff members at AKUH and NICVD. In the monthly staff meetings, we also intend to share our progress and the results with the physicians, nurses, nutritionists and other rehabilitation staff of AKUH and NICVD. This strategy will create awareness of the context-rich data and may lead to the implementation of necessary changes regarding dietary counselling and preventive measures. We the research team will make presentations at AKUH and NICVD rehabilitation centres to identify critical areas for future research. We will also share the findings with the participants and the family members to provide them with a deeper contextual understanding of the issues regarding the prevention of CVD. Participants may acknowledge the need for changing lifestyles and suggest strategies to incorporate into future preventive programmes. Healthcare teams can work very closely with all family members and address their concerns related to the family's norms and cultural values that influence the patient's lifestyle changes.

The target audience also includes administrators, clinical educators and health policymakers to inform them about preventive measures that require broader programmatic and structural changes to provide healthcare to poor people. We also plan to present our findings at one or more conferences on cardiovascular prevention at the national and international level. This research will be of great interest to cardiologists and researchers who are working in preventive cardiology, as well as some healthcare decision-makers and it will create opportunities for future interdisciplinary research.
For knowledge-translation purposes, we will also publish the study findings in highly accessed, peerreviewed scientific and health policy journals at the national and international level. This will increase the understanding of the challenges that poor people face in changing their eating behaviours, and it is possible that these contextual barriers could be included in international guidelines for CR and the secondary prevention of CVDs.

Contributors RIB drafted the manuscript. AMC and GMAH helped to refine the draft and extensively revise the manuscript. All the authors have approved the final manuscript.

Competing interests None.

Ethics approval University of Alberta, Canada and The Aga Khan University, Pakistan.

Provenance and peer review Not commissioned; internally peer reviewed.

Open Access This is an Open Access article distributed in accordance with the Creative Commons Attribution Non Commercial (CC BY-NC 3.0) license, which permits others to distribute, remix, adapt, build upon this work noncommercially, and license their derivative works on different terms, provided the original work is properly cited and the use is non-commercial. See: http:// creativecommons.org/licenses/by-nc/3.0/

\section{REFERENCES}

1. Fuster V, Kelly B. eds. Promoting cardiovascular health in the developing world. http://www.nap.edu/catalog/12815.html

2. Roger VL, Go AS, Lloyd-Jones DM, et al. Heart disease and stroke statistics-2011: a report from the American Heart Association. Circulation 2011;123:e18-209.

3. Yusuf S, Hawken S, Ônpuu S, et al. Effect of potentially modifiable risk factors associated with myocardial infarction in 52 countries (the INTERHEART Study): case-control study. Lancet 2004;364:937-52.

4. Yusuf S, Reddy S, Ounpuu S, et al. Global burden of cardiovascular diseases: part II: variations in cardiovascular disease by specific ethnic groups and geographic regions and prevention strategies. Circulation 2001;104:2855-64.

5. Reddy KS. Cardiovascular disease in developing countries: dimensions, determinants, dynamics and directions for public health action Public Health Nutrition 2006;5:231-7.

6. Nishtar S. Public health prevention of coronary heart disease in south Asia. Lancet 2002;360:1015-18.

7. Nishtar S, Voûte J. Women and heart disease: time for change. Cardiovasc Med 2007;4:231.

8. Brunner E, Rees K, Ward K, et al. Dietary advice for reducing cardiovascular risk. Cochrane Database Syst Rev 2009;(4): CD002128.

9. Olinto MTA, Gigante DP, Horta B, et al. Major dietary patterns and cardiovascular risk factors among young Brazilian adults. Eur J Nutr 2011;51:281-91.

10. Clark AM, Duncan AS, Trevoy JE, et al. Healthy diet in Canadians of low socioeconomic status with coronary heart disease: not just a matter of knowledge and choice. Heart Lung 2011;40:156-63.

11. Graham I, Atar D, Borch-Johnsen K, et al. European guidelines on cardiovascular disease prevention in clinical practice: executive summary: Fourth Joint Task Force of the European Society of Cardiology and Other Societies on Cardiovascular Disease Prevention(constituted by representatives of nine societies and by invited experts). Eur J Cardiovasc Prev Rehabil 2007;14(S):E1-40.

12. Lichtenstein $A H$, Appel LJ, Brands $M$, et al. Diet and lifestyle recommendations revision 2006: a scientific statement from the American Heart Association Nutrition Committee. Circulation 2006;114:82-96.

13. Doyle B, Fitzsimons D, McKeown $\mathrm{P}$, et al. Understanding dietary decision-making in patients attending a secondary prevention clinic following myocardial infarction. J Clin Nurs 2012;21:32-41.

14. Ristovski-Slijepcevic S, Chapman GE, Beagan BL. Engaging with healthy eating discourse(s): Ways of knowing about food and health in three ethnocultural groups in Canada. Appetite 2008;50:167-78.

15. Wetter AC, Goldberg JP, King AC, et al. How and why do individuals make food and physical activity choices? Nutr Rev 2001;59:S11-20; discussion S57-65. http://www.ncbi.nlm.nih.gov/pubmed/20946001 
16. Azad N, Nishtar S. A call for a gender specific approach to address the worldwide cardiovascular burden. Prev Control 2005;1:223-7..

17. Mendis S, Puska P, Norrving B. Global atlas on cardiovascular disease prevention and control in collaboration with the World Heart Federation and the World Stroke Organization. Geneva: World Health Organization, 2011:8-15: World Health Organization in collaboration with the World Heart Federation and the World Stroke Organization, c2011; 2011.

18. Shah $\mathrm{N}$, Ting $\mathrm{H}$, Montori $\mathrm{V}$, et al. Evidence-based therapy for myocardial infarction: medication adherence in the community. Circulation 2007;797:116-20.

19. Clark AM, DesMeules M, Luo W, et al. Socioeconomic status and cardiovascular disease: risks and implications for care. Cardiology 2009;6:712-22.

20. Block RC, Dozier AM, Hazel-Fernandez L, et al. An epidemiologic transition of cardiovascular disease risk in Carriacou and Petite Martinique, Grenada: The Grenada Heart Project. Prev Chronic Dis 2012;9:1-11.

21. Power EM. Determinants of healthy eating among low-income Canadians. Can J Public Health 2005;96(Suppl 3):S37-42, S42-8. http://www.ncbi.nlm.nih.gov/pubmed/16042163

22. Kotseva K, Wood D, De Backer G, et al. EUROASPIRE III: a survey on the lifestyle, risk factors and use of cardioprotective drug therapies in coronary patients from 22 European countries. Eur $J$ Cardiovasc Prev Rehabil 2009;16:121-37.

23. Leon AS, Franklin BA, Costa F, et al. Cardiac rehabilitation and secondary prevention of coronary heart disease: an American Heart Association scientific statement from the Council on Clinical Cardiology. Circulation 2005;111:369-76.

24. Dennis B, Aziz K, She L, et al. High rates of obesity and cardiovascula disease risk factors in lower middle class community in Pakistan: the Metroville Health Study. J Pak Med Assoc 2006;56:267-72.

25. Joshi $\mathrm{P}$, Islam $\mathrm{S}$, Pais $\mathrm{P}$, et al. Risk factors for early myocardia infarction in south Asians compared with individuals in other countries. J Am Med Assoc 2007;297:286-94.

26. Kumar BN, Selmer R, Lindman AS, et al. Ethnic differences in SCORE cardiovascular risk in Oslo, Norway. Eur J Cardiovasc Prev Rehabil 2009;16:229-34

27. Thaver I, Bhutta ZA. Poverty and ill-health: challenges, initiatives, and issues in Pakistan. http://www.sasnet.lu.se/EASASpapers/ PHDZulfiqarBhutta.pdf

28. Rosengren A, Subramanian SV, Islam S, et al. Education and risk for acute myocardial infarction in 52 high, middle and low-income countries: INTERHEART case-control study. Heart 2009;95:2014-22.

29. Bhupathiraju SN, Tucker KL. Coronary heart disease prevention: nutrients, foods and dietary patterns. Clin Chim Acta 2011;412: 1493-514.

30. Hydrie MZI, Basit A, Shera AS, et al. Dietary patterns associated with risk for metabolic syndrome in urban community of Karachi defined by cluster analysis. Pak J Nutr 2010;9:93-9.

31. Jafar TH, Qadri Z, Chaturvedi N. Coronary artery disease epidemic in Pakistan: more electrocardiographic evidence of ischaemia in women than in men. Heart 2008;94:408-13.

32. Bush J, White M, Kai J, et al. Understanding influences on smoking in Bangladeshi and Pakistani adults: community based, qualitative study. BMJ 2003;326:962-5.

33. Labonté R, Mohindra KS, Lencucha R. Framing international trade and chronic disease. Global Health 2011;7:21.

34. Appel LJ, Brands MW, Daniels SR, et al. Dietary approaches to prevent and treat hypertension: a scientific statement from the American Heart Association. Hypertension 2006;47:296-308.

35. Yusuf S, Reddy S, Ounpuu S, et al. Global burden of cardiovascular diseases: part I: general considerations, the epidemiologic transition, risk factors and impact of urbanization. Circulation 2001;104: 2746-53.

36. Ghaffar A, Kazi BM, Salman M. Health care systems in transition III: Pakistan, part I. An overview of the health care system in Pakistan. J Public Health Med 2000;22:38-42.

37. Jafar TH, Levey AS, Jafary FH, et al. Ethnic subgroup differences in hypertension in Pakistan. J Hypertension 2003;21:905-12.

38. Poverty profile: Islamic Republic of Pakistan. Japan Bank for International Cooperation [Executive summary]. October 2007. http://www.jica.go.jp/activities/issues/poverty/profile/pdf/pakistan_e.pdf

39. Haider M, West SS, Ha Q. Urbanization challenges in Pakistan: developing vision 2030. Peshawar, Pakistan: McGill University Canada \& National Institute of Infrastructure Planning Pakistan, 2006:1-9.

40. Nishtar S, Bile KM, Ahmed A, et al. Integrated population-based surveillance of noncommunicable diseases: the Pakistan model. Am J Prev Med 2005;29(5 Suppl 1):102-6.
41. Khowaja K: International perspectives. Healthcare systems and care delivery in Pakistan. J N Adm 2009;39:263-5.

42. Nishtar S. Heartfile's contribution to health systems strengthening in Pakistan. East Mediterr Health J 2006;12(Suppl 2):S38-53. http://www.ncbi.nlm.nih.gov/pubmed/17361677

43. Sabih F, Bile KM, Buehler W, et al. Implementing the district health system in the framework of primary health care in Pakistan. East Mediterr Health J 2010;16(Suppl):S132-44. http://www.ncbi.nlm.nih. gov/pubmed/21495599

44. Teo KK, Ônpuu S, Hawken S, et al. Tobacco use and risk of myocardial infarction in 52 countries in the INTERHEART study: a case-control study. Lancet 2006;368:647-58.

45. Daubenmier JJ, Weidner G, Sumner MD, et al. The contribution of changes in diet, exercise and stress management to changes in coronary risk in women and men in the multi-site cardiac lifestyle intervention program. Ann Behav Med 2007;33:57-68. http://www. ornishspectrum.com/wp-content/uploads/contribution-changes-indiet-exercise-stress.pdf

46. Thorne S, Con A, McGuinness L, et al. Health care communication issues in multiple sclerosis: an interpretive description. Qual Health Res 2004;14:5-22.

47. Hunt MR. Strengths and challenges in the use of interpretive description: reflection arising from a study of the moral experience of health professionals in humanitarian work. Qual Health Res 2009;19:1284-92.

48. Cohen DJ, Crabtree BJ. Evaluative criteria for qualitative research in health care: controversies and recommendations. Ann Family Med 2008;6:331-9.

49. Lincoln YS, Guba EG. Naturalistic inquiry. Beverly Hills, CA: Sage, 1985:14-90.

50. Thorne S, Reimer Kirkham S, O'Flynn-Magee K. The analytic challenge in interpretive description. Int J Qual Methods 2004;3:1-21.

51. Higginbottom GMA. Sampling issues in qualitative research. Nurse Res 2004;12:7-19. http://www.ncbi.nlm.nih.gov/pubmed/ 15493211

52. Clark AM, Lissel S, Davis C. Complex critical realism: tenets and application in nursing research. Adv Nurs Sci 2008;31:E67-79.

53. Bhaskar R. A realist theory of science. Brighton, UK: Harvester, 1975:258-92.

54. Wilson V, McCormack B. Critical realism as emancipatory action: the case for realistic evaluation in practice development. Nurs Phil 2006;7:45-57

55. Howard BV, Van Horn L, Hsia J, et al. Low-fat dietary pattern and risk of cardiovascular disease: the Women's Health Initiative Randomized Controlled Dietary Modification Trial. J Am Med Assoc 2006;295:655-66.

56. Population estimates of Pakistan. United Nations urbanization report [Prospects Report]. March 2010. http://www.un.org/esa/population

57. Marsh DR, Kadir MM, Husein K, et al. Adult mortality in slums of Karachi, Pakistan. J Pak Med Assoc 2000;50:300-6. http://www. ncbi.nlm.nih.gov/pubmed/11043020

58. National Institute of Cardiovascular Diseases: About us. http://www. nicvd.edu.pk/AboutUs.htm

59. National Institute of Cardiovascular Diseases: Facilities: Clinical facilities. http://www.nicvd.edu.pk/Patient.htm

60. National Institute of Cardiovascular Diseases: Facilities: Department of Rehabilitation and Preventive Cardiology. http://www.nicvd.edu.pk/ Rehabilitation.htm

61. Aga Khan University Hospital: About us. http://hospitals.aku.edu/ karachi/aboutus/Pages/home.aspx

62. Nishtar S, Khalid F, Ikram A, et al. Protecting the poor against health impoverishment in Pakistan: Proof of concept of the potential within innovative web and mobile phone technologies. World Health Report. 2010. http://www.who.int/healthsystems/topics/research/ 55Heartfile HEF POC.pdf

63. Thorne S, Reimer Kirkham S, Macdonald-Emes J. Focus on qualitative methods interpretive description:a noncategorical qualitative alternative for developing nursing knowledge. Qual Res 1997;20:169-77.

64. Harwood L, Clark AM. Understanding health decisions using critical realism: home-dialysis decision-making during chronic kidney disease. Nurs Inquiry 2011;19:29-38.

65. ATLAS.ti: Home http://www.atlasti.com/index.htm

66. Miles MB, Huberman AM, Saldana J. Qualitative data analysis. 3rd edn. Thousand Oaks, CA: Sage, 2013:69-211.

67. Higginbottom GM, Vallianatos H, Forgeron J, et al. Food choices and practices during pregnancy of immigrant and Aboriginal women in Canada: a study protocol. BMC Pregnancy Childbirth 2011;11:100. 